\title{
Practical management of toxicities associated with targeted therapies for advanced gastroenteropancreatic neuroendocrine tumors
}

\author{
Pieter-Jan Cuylea, Hans Prenen ${ }^{b}$ \\ Imelda General Hospital, Bonheiden; University Hospitals Gasthuisberg Leuven, Leuven, Belgium
}

\section{Abstract}

\section{Introduction}

Neuroendocrine neoplasms (NENs) are a heterogeneous group of tumors arising from the diffuse endocrine system with a diverse range of functional and behavioral characteristics. NENs most frequently originate within the gastroenteropancreatic system (60-70\%) and bronchopulmonary tract (25\%). They are historically regarded as infrequent malignancies, with an incidence of 2.53/100,000/ year as reported by the RARECARE working group [1]; however, both incidence and prevalence appear to be rising in recent decades $[2,3]$. The 2017 WHO classification of tumors of endocrine organs has recently been published to provide us

Department of Gastroenterology/Digestive Oncology, a Imelda General Hospital, Bonheiden (Pieter-Jan Cuyle); ${ }^{b}$ University Hospitals Gasthuisberg Leuven, Leuven (Hans Prenen), Belgium

Conflict of Interest: Pieter-Jan Cuyle: travel grant from Ipsen, consultancy honoraria from Novartis. Hans Prenen: honoraria and/or travel grants from Ipsen, Novartis and Pfizer. Hans Prenen is supported by the Belgian Foundation against Cancer

Correspondence to: Pieter-Jan Cuyle, Imeldaziekenhuis Bonheiden, Imeldalaan 9, 2820 Bonheiden, Belgium,

e-mail: pieter-jan.cuyle@imelda.be

Received 8 October 2017; accepted 13 November 2017; published online 8 January 2018

DOI: https://doi.org/10.20524/aog.2018.0224 with an update on the classification of pancreatic NENs [4], but it is likely to be implemented in all NENs in the near future. It now splits up NENs according to histological features and measurement of a proliferative marker index, the Ki-67 (\%). Well-differentiated neuroendocrine tumors (NETs) are clearly distinguished from poorly-differentiated neuroendocrine carcinomas (NECs), the latter being characterized by a smallcell or large-cell histological pattern, aggressive clinical behavior and a very high Ki-67, usually around 70\%. Welldifferentiated NETs are further subdivided according to the Ki-67 into low-grade G1 tumors (Ki-67 <3\%), intermediategrade G2 (Ki-67 between 3-20\%) and a new subgroup, the well-differentiated high-grade G3 NETs with a Ki-67 >20\%, often around $40 \%$.

The most common primary sites for gastroenteropancreatic neuroendocrine tumors (GEPNETs) are stomach, appendix, small intestine, rectum, pancreas and colon. Patients may present either with symptoms provoked by hormonal hypersecretion (30\% of cases) or, if non-functional, by symptoms of local growth (mass, obstruction, or bleeding). The most prevalent functional syndrome is the carcinoid syndrome, covering a spectrum of symptoms such as diarrhea, flushing, wheezing and valvular heart disease (carcinoid heart disease). Extension beyond the primary tumor usually involves locoregional lymph nodes and more than half of the patients present with distant metastases at diagnosis. The most frequent metastatic site is the liver, followed by peritoneal, bone, lung, and brain metastases. Rarely, metastases can be seen in the heart, the breast, or the skin. 


\section{Balancing risks and benefits in GEPNET treatment}

Thorough clinical assessment (symptoms, potential distinct functional syndromes, patient characteristics), together with primary tumor location and delineation of the tumor's grade and TNM stage as well as its somatostatin receptor (SSTR) expression profile, will guide treatment decision-making in these patients. In the advanced setting, treatment aims at controlling tumor growth and symptoms, while preserving and improving quality of life. As this disease may be relatively slow-growing, patients are expected to be treated for longer periods; thus, even mild toxicities can influence quality of life, compliance and outcome in the long run.

Surgery represents the only potentially curative option in this disease. Aggressive surgical strategies, such as en-bloc resection of primary tumor and metastases or even liver transplantation in highly selected cases, are to be considered when judging therapeutic options. Locoregional techniques, such as ablation, chemo- and/or radio-embolization and stereotactic body radiation therapy are often applied to treat metastases.

Current targeted therapy options for advanced GEPNETs include somatostatin analogues (SSAs), interferon (IFN)- $\alpha$, peptide receptor radionucleotide therapy (PRRT) and the molecular targeted agents everolimus and sunitinib. Remarkably, none of these systemic therapies can be graded as meaningfully clinically beneficial according to the European Society for Medical Oncology Magnitude of Clinical Benefit Scale (ESMO-MCBS) [5].

This review summarizes practical recommendations for toxicity management associated with these treatments. Cytotoxic chemotherapy remains the mainstay of advanced NEC treatment and can be a treatment option in advanced pancreatic NETs (pNETs); however, its specific adverse event management lies beyond the scope of this article.

\section{SSAS}

Most NETs express several SSTRs at high levels, with SSTR2 acting as the predominant subtype [6]. However expression levels and predominant subtype can vary between NET tumor types [7]. Somatostatin is a natural polypeptide playing an inhibitory role in pituitary, pancreatic and gastrointestinal (GI) hormone secretion, through its high affinity binding to all five SSTR subtypes (SSTR1-5). Its therapeutic use is limited by a very short circulation half-life of around $2 \mathrm{~min}$. This led to the development of synthetic SSAs with longer half-lives, initially intended to palliate hormonal symptoms in functional GEPNETs.

Octreotide and lanreotide are synthetic octapeptides with high affinity for SSTR2 and moderate affinity for SSTR5. Octreotide was the first to be approved in 1988 for the treatment of hormonal syndromes and has a half-life of 1.5-2 h. It needs to be injected subcutaneously at a dose of 150-500 $\mu \mathrm{g}$, 3-4 times a day. Octreotide long-acting repeatable (LAR) depot formulation is a slow-release product incorporating octreotide in microspheres of biodegradable polymer. Octreotide LAR
$(10,20$, or $30 \mathrm{mg})$ is typically administered intramuscularly every 4 weeks. Likewise, lanreotide has a depot formulation, called lanreotide autogel, available in 60,90 and $120 \mathrm{mg}$, administered deep subcutaneously every 4 weeks. Both octreotide and lanreotide have shown comparable efficacy with regard to symptomatic control rates for carcinoid syndrome in $60-72 \%$ and $55-75 \%$ of cases, respectively [8-11].

As suggested in preclinical studies, SSAs also exhibit antiproliferative activity in NETs, which is more difficult to explain than their antisecretory effects. SSTR activation on the tumor cells might trigger direct antiproliferative mechanisms through the PI3K/Akt/mTOR pathway, inducing tumor suppressor gene expression, cell cycle arrest and apoptosis [12]. Indirect antiproliferative effects through inhibition of growth factor secretion and antiangiogenic activity have also been suggested [13]. A significant antitumor activity of SSAs was documented in two phase 3 , prospective, randomized, placebo-controlled trials. The PROMID trial established the efficacy of octreotide LAR $30 \mathrm{mg}$ every 4 weeks in inoperable or metastatic, functional or non-functional, well-differentiated G1 midgut NETs, especially in patients with resected primary and low hepatic tumor burden $[14,15]$. The more recent CLARINET study clearly demonstrated the antiproliferative effect of lanreotide autogel $120 \mathrm{mg}$ every 4 weeks in inoperable or metastatic, non-functional, well-differentiated G1 and G2 (Ki-67 <10\%) GEPNETs, regardless of tumor burden [16]. Both depot formulations, octreotide LAR and lanreotide autogel, are currently the most used SSAs in clinical practice.

With over 25 years of clinical experience with SSAs, it is safe to state that they are generally well tolerated. GI side effects (diarrhea, steatorrhea, abdominal cramps, flatulence, and nausea) are reported most frequently, but tend to be mild to moderate in severity. Patients should be informed that GI toxicity often decreases in intensity over time or resolves spontaneously during further treatment. Supportive measures should be taken and in case of overt steatorrhea, pancreatic enzymes should be substituted. Altered cholecystokinin secretion causes cholelithiasis in up to $50 \%$ of SSA treated GEPNET patients, which can be complicated by bilious attacks, acute cholecystitis or biliary pancreatitis occurrence [17]. Therefore, prophylactic cholecystectomy can be considered in patients receiving SSAs. Octreotide and lanreotide have the potential to alter glucose homeostasis and cause hyperglycemia; however, their exact impact remains debatable. Patients with known glucose intolerance or diabetes mellitus should be instructed to maintain strict blood glucose monitoring nevertheless. Rare side effects include hypoglycemia, hair loss, hypothyroidism, headache, myalgia, acute hepatitis, hyperbilirubinemia, site injection reaction, obstipation, paralytic ileus, bradycardia and QTc prolongation [6]. Higher doses of octreotide LAR (>30 mg/4 weeks) are frequently used in clinical practice to treat refractory carcinoid syndrome symptoms based on small retrospective and prospective studies [18]. Interestingly, none of these studies reported increased toxicity with the use of a higher dosage or a decreased dosing interval [19].

Pasireotide (SOM230) is a next-generation SSA with high affinity for SSTR1-3 and SSTR5, available in a short acting subcutaneous form and an LAR form, injected intramuscularly 
every 4 weeks. Its place in GEPNET treatment is currently unclear after a randomized phase 3 trial failed to prove an advantage in carcinoid symptom control when compared to high-dose octreotide LAR [20]. Moreover, despite a safety profile similar to that of first-generation SSAs in general, pasireotide induces a higher frequency and degree of hyperglycemia (11 vs. $0 \%$ ) [20].

\section{IFN-a}

IFNs are cytokines mediating antiviral, antiproliferative and antitumor activities. IFN- $\alpha$ has been used for the treatment of certain solid malignancies, such as melanoma and NETs; however, its mode of action is complex and remains to be further elucidated. It targets tumor cells directly, inducing cell cycle arrest and apoptosis on the one hand and acting through immunomodulation and antiangiogenesis on the other [6]. In the GEPNET indication, IFN- $\alpha$ is usually given as a subcutaneous injection at a dose of 3-5 million units three times a week or, alternatively, as weekly injections of 50-180 $\mu \mathrm{g}$ long-acting pegylated IFN- $\alpha$. Because of its unfavorable toxicity profile, IFN- $\alpha$ is rarely used as first-line therapy (except in case of SSTR negative NETs [21]) and is mainly used as an additive to other treatment or as bridging while waiting for initiation of other therapy, such as embolization or PRRT. According to ENETS guidelines, IFN- $\alpha$ is an established and approved second-line (add-on) therapy for refractory carcinoid syndrome or functional pNETs [22]. It can also be considered as an antiproliferative therapeutic option in GEPNETs; however, prospective data are scarce and largely inconclusive $[6,22]$.

Compared to SSAs, IFN- $\alpha$ is associated with more side effects, some of them occurring early on during the induction phase (i.e., flu-like symptoms, neutropenia, thrombocytopenia), while others are linked to a prolonged duration of therapy (i.e., fatigue, depression/anxiety). Routine laboratory monitoring is recommended before the start, after 2, 4 and 12 weeks, and every 3 months thereafter (complete blood count with differential, creatinine, electrolytes, liver function enzymes, creatinine kinase, thyroid stimulating hormone, triglycerides, and serum glucose) [23]. Recommendations for toxicity management are summarized in Table 1 [6,23-25].

\section{Telotristat ethyl}

Although SSAs achieve a reasonably high rate of symptom control in carcinoid syndrome, around $20 \%$ of patients suffer from persistent and debilitating symptoms $[9,26]$. In these refractory cases high doses of SSAs, association of IFN-a or switching of SSAs are often attempted, along with efforts to reduce tumor burden. Telotristat ethyl, a first-in-class, smallmolecule, oral tryptophan hydroxylase inhibitor, suppresses the rate-limiting step in serotonin production. The drug has been approved by the Food and Drug Administration (FDA) and European Medicine Agency (EMA) at a dose of $250 \mathrm{mg}$ t.i.d. for the treatment of SSA-refractory carcinoid syndrome and is given in addition to the SSA administration [27]. The efficacy of telotristat ethyl in diarrhea reduction in this setting has been documented in two double-blind pivotal phase 3 trials, TELESTAR and TELECAST, and the clinical benefit was maintained over the longer term in open-label extension studies [28-31]. These trials were not powered to assess other carcinoid syndrome symptoms and the reported reductions in abdominal pain and flushing were not statistically significant. The influence of telotristat ethyl on carcinoid heart disease and mesenteric fibrosis development and progression remains to be studied. The safety profile is very favorable, with very few side effects, but data from long-term follow-up trials, such as TELEPATH (NCT02026063), need to be awaited. Among the adverse events of special interest (depression related, elevated hepatic enzymes, GI symptoms), slightly higher rates of nausea, constipation and depression were reported in the treatment arms when compared to the placebo arms [27,32]. Despite initial concern about the potential impact of long-term serotonin synthesis inhibition, no depression-related serious adverse events have been reported.

\section{Everolimus}

The PI3K/AKT/mTOR signaling pathway plays a pivotal role in recognition of stress signals and regulation of cell survival, proliferation and apoptosis, and it is deregulated in several human malignancies, including NETs. Everolimus is a rapamycin analog that inhibits the multiprotein complex mTOR complex 1 (mTORC1). Oral everolimus at a daily dose of $10 \mathrm{mg}$ achieves peak concentration after 1-2 $\mathrm{h}$ and reaches a steady state condition within 7 days. The drug has been extensively studied in NET treatment, either alone or in combination. Everolimus is considered a valid treatment option in NETs based on two phase 3 registry trials. The RADIANT-3 trial conducted by Yao et al, in unresectable or metastatic, well-differentiated (G1-G2) progressive pNETs, showed a significantly longer median progression-free survival (PFS) for everolimus compared to placebo (11.4 vs. 5.4 months; hazard ratio $[\mathrm{HR}]$ 0.34, $\mathrm{P}<0.0001$ ) [33]. Quite similarly, RADIANT-4, a large placebo-controlled study that used everolimus monotherapy to treat unresectable or metastatic well-differentiated (G1-G2) non-functional GI or lung NETs, demonstrated a statistically significant median PFS benefit for everolimus in this population (11 vs. 3.9 months; HR 0.48 , $\mathrm{P}<0.00001)$ [34]. This drug seems to induce tumor stabilization rather than regression, as conventional tumor response rates per Response Evaluation Criteria In Solid Tumors (RECIST) are rarely noted ( $<5 \%$ of patients). It is common practice to combine everolimus with SSAs, especially in functional NETs. However, superiority of the combination over everolimus monotherapy in terms of antiproliferative effect has never been clearly demonstrated.

Class effects of mTOR inhibitors include epithelialcutaneous toxicity (i.e., stomatitis and rash), interstitial lung 
Table 1 Recommendations for clinical management of interferon (IFN)- $\alpha$ toxicity

\begin{tabular}{|c|c|c|}
\hline General description/incidence (\%) & Preventive measures & Treatment \\
\hline $\begin{array}{l}\text { Flu-like symptoms (not available) } \\
\text { - headaches, myalgia, fever, nausea, } \\
\text { diarrhea } \\
\text { - onset 3-5 h after subcutaneous } \\
\text { administration } \\
\text { - incidence decreases after } 4 \text { weeks of } \\
\text { therapy }\end{array}$ & $\begin{array}{l}\text { - premedication with antipyretics and } \\
\text { analgesics } \\
\text { - education on maintaining adequate } \\
\text { hydration } \\
\text { - administration in the evening (sleep } \\
\text { through most of these symptoms) }\end{array}$ & $\begin{array}{l}\text { - paracetamol up to } 4 \mathrm{~g} / \text { day, starting } 30 \text { min before } \\
\text { - antiemetics can reduce nausea, benzodiazepines } \\
\text { in refractory cases, corticosteroids to be } \\
\text { avoided (counteractive to IFN- } \alpha \text { as an } \\
\text { immunosuppressant) } \\
\text { - loperamide or similar agents for diarrhea }\end{array}$ \\
\hline Anorexia (not available) & $\begin{array}{l}\text { - patient education on ideal body } \\
\text { weight, optimal calorie-dense diet and } \\
\text { maintaining adequate hydration } \\
\text { - smaller, more frequent meals, high } \\
\text { protein supplement }\end{array}$ & - consider treatment interruption in severe cases \\
\hline $\begin{array}{l}\text { Hepatotoxicity ( } 30 \%) \\
\text { - rule out other causes/underlying } \\
\text { conditions }\end{array}$ & - avoid hepatotoxic agents & $\begin{array}{l}\text { - grade } 3 / 4 \text { : interrupt until recovery to } \leq \text { grade } 1 / 2 \\
\text { - stepwise dose reduction on reinitiation }\end{array}$ \\
\hline $\begin{array}{l}\text { Thyroid dysfunction ( } 8-20 \%) \\
\text { - hyperthyroidism followed by } \\
\text { hypothyroidism (auto-immune pattern; } \\
\text { thyroid antibodies often detectable) }\end{array}$ & & $\begin{array}{l}\text { - beta-blockers, thyroid inhibitors or thyroxine } \\
\text { replacement therapy per standard guidelines } \\
\text { - IFN- } \alpha \text { dose interruption or adjustment exceptional }\end{array}$ \\
\hline $\begin{array}{l}\text { Fatigue (not available) } \\
\text { - rule out other causes (thyroid } \\
\text { dysfunction, psychological/emotional } \\
\text { distress, anemia, etc.) }\end{array}$ & $\begin{array}{l}\text { - ensure a consistent sleep cycle } \\
\text { - maintain activity levels during the } \\
\text { day } \\
\text { - avoid excessive caffeine and alcohol } \\
\text { - adequate fluid and nutritional } \\
\text { intake }\end{array}$ & $\begin{array}{l}\text { - in analogue to cancer-related fatigue guidelines } \\
\text { - cognitive and behavioral sleep therapy }\end{array}$ \\
\hline $\begin{array}{l}\text { Hematologic toxicity } \\
\text { Anemia }(25 \%) \\
\text { Thrombocytopenia }(10-20 \%) \\
\text { Leukopenia }(40-60 \%)\end{array}$ & & $\begin{array}{l}\text { - thrombocytopenia }<50 \times 10^{9} / \mathrm{L}: 50 \% \text { dose reduction } \\
\text { - thrombocytopenia }<25 \times 10^{9} / \mathrm{L} \text { : discontinue therapy } \\
\text { - neutropenia }<0.75 \times 10^{9} / \mathrm{L}: 50 \% \text { dose reduction } \\
\text { - neutropenia }<0.5 \times 10^{9} / \mathrm{L} \text { : discontinue therapy }\end{array}$ \\
\hline Hypertriglyceridemia (not available) & $\begin{array}{l}\text { - limit dietary intake of saturated fat, } \\
\text { cholesterol, simple sugars and alcohol, } \\
\text { stimulate intake of soluble fiber and } \\
\text { plant sterols } \\
\text { - reduce weight and increase physical } \\
\text { activity in overweight patients }\end{array}$ & $\begin{array}{l}\text { - treat dyslipidemia according to standard guidelines } \\
\text { - prompt initiation of fibrates if triglycerides } \\
>500 \mathrm{mg} / \mathrm{dL} \text { (acute pancreatitis risk) }\end{array}$ \\
\hline $\begin{array}{l}\text { Neuropsychiatric (not available) } \\
\text { - depression, sleeplessness, irritability, } \\
\text { concentration difficulty, anxiety }\end{array}$ & $\begin{array}{l}\text { - educate patients and caregivers to } \\
\text { report symptoms } \\
\text { - psychiatric advice in advance in case } \\
\text { of psychiatric disorder history }\end{array}$ & $\begin{array}{l}\text { - zolpidem/zoplicone } 1^{\text {st }} \text { choice for sleeplessness } \\
\text { - selective serotonin reuptake inhibitor } 1^{\text {st }} \text { choice for } \\
\text { depression; refer to psychiatrist; cognitive therapy } \\
\text { - psychostimulants/anxiolytics as indicated } \\
\text { - treat } \geq 4 \text { weeks after IFN cessation ( } 3 \text { months in PEG) } \\
\text { - dose modification or interruption in severe cases }\end{array}$ \\
\hline $\begin{array}{l}\text { Cutaneous (not available) } \\
\text { - local reactions injection site, xerosis } \\
\text { cuti, sicca symptoms, pruritus, alopecia }\end{array}$ & & $\begin{array}{l}\text { - urea-containing emollients } \\
\text { - artificial tears/saliva } \\
\text { - estriol crème/lubricants }\end{array}$ \\
\hline
\end{tabular}

disease (non-infectious pneumonia), metabolic disturbances and immune suppression. Other common side effects are nausea, diarrhea, fatigue and peripheral edema [33-35]. About $5-7 \%$ of patients experience grade 3-4 toxicity. Most classeffect adverse events are manageable and resolve without the need for treatment discontinuation. According to the everolimus prescribing information, a number of parameters should be measured at baseline and followed during treatment (Table 2) [36]. Clinical guidance on toxicity management
Table 2 Follow-up recommendations under everolimus therapy

At baseline and periodically thereafter

- physical examination

- urine dipstick for proteinuria

- complete blood count with differential

- blood chemistry including creatinine, electrolytes, liver function

- blood cholesterol and triglycerides

- blood glucose*

${ }^{*}$ weekly during the first month of treatment in high risk patients 
Table 3 Recommendations for clinical management of everolimus toxicity

\begin{tabular}{|c|c|c|}
\hline General description/incidence (\%) & Preventive measures & Treatment \\
\hline $\begin{array}{l}\text { Stomatitis }(62-64 \%) \\
\text { - mTOR-inhibitor associated } \\
\text { stomatitis (mIAS) differs from } \\
\text { chemotherapy/radiation induced stomatitis } \\
\text { - "aphthous-like"; well circumscribed } \\
\text { single or multiple ovoid-shaped ulcerations } \\
\text { typically on non-keratinized mucosa. } \\
\text { Painful, long-lasting and more functionally } \\
\text { limiting than their small size }(<0.5 \mathrm{~cm}) \\
\text { would suggest } \\
\text { - lesions typically present within first } \\
\text { treatment cycle } \\
\text { - most common dose limiting toxicity } \\
\text { - specific pathophysiology largely unknown }\end{array}$ & $\begin{array}{l}\text { - patient education on prompt symptom } \\
\text { reporting } \\
\text { - basic oral hygiene and dental } \\
\text { examinations } \\
\text { - avoid epithelial injury } \\
\text { - avoiding spicy, acidic, salty, crunchy } \\
\text { food } \\
\text { - avoiding alcohol- or peroxide } \\
\text { containing mouthwash } \\
\text { - brushing with soft toothbrush } \\
\text { - frequent bland rinses with sterile water } \\
\text { or saline } \\
\text { - sodium bicarbonate rinses and antiseptic } \\
\text { mouthwashes lack benefit or have } \\
\text { produced inconsistent results }\end{array}$ & $\begin{array}{l}\text { - evaluate for bacterial, viral or fungal } \\
\text { infections } \\
\text { - topical and systemic pain } \\
\text { control (non-opioid analgesics often } \\
\text { insufficient) } \\
\text { - topical or intralesional corticosteroids } \\
\text { - e.g., dexamethasone } 0.5 \mathrm{mg} / 5 \mathrm{~mL} \text { oral } \\
\text { solution } \\
\text { - e.g., prednisolone } 15 \mathrm{mg} / 5 \mathrm{~mL} \text { oral } \\
\text { solution } \\
\text { - systemic corticosteroids for severe } \\
\text { persisting mIAS } \\
\text { - e.g., prednisone } 5 \mathrm{mg} \text { bid } \\
\text { - grade 2/3: dose interruption until recovery } \\
\text { to } \leq \text { grade } 1 \\
\text { - grade 2: reinitiate at same dose, unless } \\
\text { recurrent } \\
\text { - grade } 3 \text { : reinitiate at lower dose } \\
\text { - grade } 4 \text { : permanent everolimus } \\
\text { discontinuation }\end{array}$ \\
\hline $\begin{array}{l}\text { Pneumonitis (8-17\%) } \\
\text { - inconsistent definition and reporting in } \\
\text { literature } \\
\text { - often asymptomatic or mildly } \\
\text { symptomatic (cough, dyspnea, hypoxia, } \\
\text { pleural effusion) } \\
\text { - potentially life-threatening (respiratory } \\
\text { insufficiency) } \\
\text { - non-specific clinical, radiological, } \\
\text { pathological features } \\
\text { - wide range of onset over several months } \\
\text { - diagnostic work-up (high-resolution } \\
\text { computed tomography, pulmonary } \\
\text { function test, often broncho-alveolar } \\
\text { lavation) to differentiate from } \\
\text { infectious (incl. opportunistic) or other } \\
\text { cardiopulmonary events }\end{array}$ & $\begin{array}{l}\text { - patient education on prompt symptom } \\
\text { reporting } \\
\text { - everolimus should not be used in severe } \\
\text { pre-existent pulmonary disease }\end{array}$ & $\begin{array}{l}\text { - grade } 1 \text { : watchful waiting } \\
\text { - grade } 2 \text { : } \\
\text { - consider dose interruption until recovery } \\
\text { to } \leq \text { grade } 1 \\
\text { - reinitiate at lower dose } \\
\text { - discontinue if recovery takes }>4 \text { weeks } \\
\text { - grade } 3 \text { : } \\
\text { - dose interruption until recovery to } \leq \\
\text { grade } 1 \\
\text { - reinitiate at lower dose, unless recurrent } \\
\text { - grade } 4 \text { : permanent everolimus } \\
\text { discontinuation } \\
\text { - systemic corticosteroids to be considered } \\
\text { for } \geq \text { grade } 2 \\
\text { - e.g., prednisone } 40 \text { mg q.d.; dose temper } \\
\text { over several weeks; add pneumocystis } \\
\text { prophylaxis during treatment }\end{array}$ \\
\hline $\begin{array}{l}\text { Rash }(27-49 \%) \\
\text { - dermatitis presents as maculopapular or } \\
\text { papulopustular eruptions, usually on upper } \\
\text { trunk, face, scalp, neck and sometimes } \\
\text { extremities } \\
\text { - starting within the first month of } \\
\text { treatment }\end{array}$ & $\begin{array}{l}\text { - prevent skin irritation and dryness } \\
\text { - avoid detergents, disinfectants, soap } \\
\text { - avoid hot showers and excessive sun } \\
\text { exposure } \\
\text { - use pH-neutral, fragrance-free skin } \\
\text { care products } \\
\text { - topical application of (urea-based) } \\
\text { moisturizers. }\end{array}$ & $\begin{array}{l}\text { - majority resolves without therapeutic } \\
\text { interventions } \\
\text { - grade } 1 / 2 \text { : topical corticosteroids and } \\
\text { moisturizer } \\
\text { - consider dose interruption until recovery } \\
\text { to } \leq \text { grade } 1 \\
\text { - reinitiate at same dose, unless recurrent } \\
\text { - grade } 3 \text { : topical and/or systemic } \\
\text { corticosteroids } \\
\text { - dose interruption until recovery to } \\
\text { sgrade } 1 \\
\text { - reinitiate at lower dose, discontinue if } \\
\text { grade } 3 \text { recurs } \\
\text { - grade } 4 \text { : permanent everolimus } \\
\text { discontinuation }\end{array}$ \\
\hline
\end{tabular}


Table 3 (Continued)

\begin{tabular}{|c|c|c|}
\hline General description/incidence (\%) & Preventive measures & Treatment \\
\hline $\begin{array}{l}\text { Metabolic events } \\
\text { Hyperglycemia (10-15\%) }\end{array}$ & $\begin{array}{l}\text { - everolimus should not be used in } \\
\text { uncontrolled diabetes }\end{array}$ & $\begin{array}{l}\text { - treat hyperglycemia according to standard } \\
\text { guidelines }\end{array}$ \\
\hline \multirow[t]{4}{*}{ Dyslipidemia (not available) } & $\begin{array}{l}\text { - optimization of lipidemic control (serum } \\
\text { cholesterol }<300 \mathrm{mg} / \mathrm{dL} \text { and fasting } \\
\text { triglycerides } \leq 2.5 \times \text { upper limit }\end{array}$ & $\begin{array}{l}\text { - treat dyslipidemia according to standard } \\
\text { guidelines }\end{array}$ \\
\hline & \multirow{3}{*}{$\begin{array}{l}\text { - limit dietary intake of saturated fat, } \\
\text { cholesterol, simple sugars and alcohol, } \\
\text { stimulate intake of soluble fiber and plant } \\
\text { sterols } \\
\text { - reduce weight and increase physical } \\
\text { activity in overweight patients }\end{array}$} & $\begin{array}{l}\text { - grade } 3 \text { : } \\
\text { - dose interruption until recovery to } \\
\text { sgrade } 1\end{array}$ \\
\hline & & $\begin{array}{l}\text { - reinitiate at lower dose } \\
\text { - grade 4: permanent everolimus } \\
\text { discontinuation }\end{array}$ \\
\hline & & $\begin{array}{l}\text { - prompt initiation of fibrates if triglycerides } \\
>500 \mathrm{mg} / \mathrm{dL} \text { (acute pancreatitis risk) }\end{array}$ \\
\hline \multirow{7}{*}{$\begin{array}{l}\text { Infections (20-29\%) } \\
\text { - localized and systemic } \\
\text { infections (bacterial, viral, candidiasis, } \\
\text { invasive fungal infections) }\end{array}$} & \multirow{7}{*}{$\begin{array}{l}\text { - screening for tuberculosis and } \\
\text { hepatitis B virus status in high prevalence } \\
\text { areas }\end{array}$} & \multirow{2}{*}{$\begin{array}{l}\text { - prompt diagnosis and } \\
\text { treatment (antibiotic, antifungal or antiviral } \\
\text { treatment as appropriate) }\end{array}$} \\
\hline & & \\
\hline & & $\begin{array}{l}\text { - grade } 2 / 3 \text { : dose interruption until recovery } \\
\text { to } \leq \text { grade } 1\end{array}$ \\
\hline & & $\begin{array}{l}\text { - grade 2: reinitiate at same dose, unless } \\
\text { recurrent }\end{array}$ \\
\hline & & - grade 3: reinitiate lower dose \\
\hline & & discontinue if grade 3 recurs \\
\hline & & $\begin{array}{l}\text { - grade 4: permanent everolimus } \\
\text { discontinuation }\end{array}$ \\
\hline Hematologic toxicity & & - grade $2 / 3$ : dose interruption until recovery \\
\hline Anemia $(15-17 \%)$ & & to $\leq$ grade 1 \\
\hline Thrombocytopenia (13-14\%) & & $\begin{array}{l}\text { - grade 2: reinitiate at same dose, unless } \\
\text { recurrent }\end{array}$ \\
\hline & & $\begin{array}{l}\text { - grade 3: reinitiate lower dose } \\
\text { discontinue if grade } 3 \text { recurs }\end{array}$ \\
\hline \multirow[t]{5}{*}{ Neutropenia (not available) } & & $\begin{array}{l}\text { - grade 4: permanent everolimus } \\
\text { discontinuation }\end{array}$ \\
\hline & & $\begin{array}{l}\text { - grade 3: dose interruption until recovery } \\
\text { to } \leq \text { grade } 1\end{array}$ \\
\hline & & reinitiate at same dose, unless recurrent \\
\hline & & $\begin{array}{l}\text { - grade } 4 \text { : dose interruption until recovery } \\
\text { to } \leq \text { grade } 1 \\
\text { reinitiate lower dose }\end{array}$ \\
\hline & & discontinue if grade $3 / 4$ recurs \\
\hline
\end{tabular}

is summarized in Table 3 [33-41]. Dose reduction usually involves a switch to a daily dose of $5 \mathrm{mg}$. In their meta-analysis, Ravaud et al found a convincing correlation between a higher everolimus exposure and improved tumor size reduction on the one hand, but an increased risk of $\geq$ grade 3 pulmonary, stomatitis and metabolic events on the other [42]. Caution is warranted when prescribing systemic corticosteroids for treatment of everolimus-associated adverse events, in view of the additional immunosuppressive effect and the possible interaction with CYP3A4, which can lead to reduced everolimus efficacy.

\section{Sunitinib}

Sunitinib malate is an oral multitargeted tyrosine kinase inhibitor (MTKI) that blocks, among others, the vascular endothelial growth factor receptor, platelet-derived growth factor receptor and c-KIT receptor. It has gained approval for the treatment of unresectable or metastatic, well-differentiated, progressive pNETs, based on the phase III trial by Raymond et al, which demonstrated a robust median PFS benefit of continuous daily dosing (CCD) with sunitinib $37.5 \mathrm{mg} /$ day over placebo $[43,44]$. Similarly to everolimus treatment, objective 
responses according to RECIST are exceptional under sunitinib treatment. The CCD administration schedule differs from its use in advanced renal cell carcinoma (RCC) and imatinib-resistant GI stromal tumors, in which intermittent dosing at $50 \mathrm{mg} /$ day, 4 weeks on and 2 weeks off, is used. A meta-analysis of sunitinib data in solid tumor trials has shown a clear association between higher sunitinib exposure and improvement of outcome parameters [45]. These findings stress the importance of maintaining dose intensity and avoiding unnecessary dose reductions or delays by early and adequate toxicity prevention and management. Most common adverse events are grade 1-2 and include fatigue, palmar-plantar erythrodysesthesia (hand-foot syndrome; HFS), hypertension, GI symptoms and hypothyroidism. Grade 3-4 toxicity occurs in 5\% of cases, with neutropenia (12\%) and hypertension (10\%) as most frequent events; however, there have been no reports of febrile neutropenia so far [44]. MTKI-associated HFS has certain distinct clinical and histopathological features, likely due to different pathophysiology, differentiating this entity from chemotherapyinduced (e.g. fluoropyrimidines) HFS [46]. Typical hair color changes and skin depigmentation are quite harmless and occur in $29 \%$ of treated patients [44]. Some adverse events have been studied as surrogate biomarkers of sunitinib efficacy in RCC treatment; however, their impact on pNET management remains to be clarified [47]. According to the sunitinib prescribing information, a number of parameters should be measured at baseline and followed during treatment (Table 4) [48]. Clinical guidance on toxicity management is summarized in Table $5[43,44,49]$. Dose adjustments should be made in $12.5 \mathrm{mg}$ increments and in some cases an intermittent dosing schedule (e.g. 2 weeks on/1 week off) might be appropriate [48].

\section{PRRT}

PRRT is a novel form of targeted systemic radiotherapy delivering radionucleotides to tumor cells expressing high levels of SSTRs. This strategy involves a SSA carrier molecule (octreotide or octreotate) attached to a radionucleotide by a chelator, of which DOTA (tetraazacyclododecane-tetraacetic acid) and DTPA (diethylenetriamine-pentaacetic acid) are the most commonly used. Frequently used radionucleotides include yttrium-90 ( ${ }^{90} \mathrm{Y}$-dotatoc) and lutetium-177 $\left({ }^{177} \mathrm{Lu}-\right.$ dotatate). Both ${ }^{90} \mathrm{Y}$ and ${ }^{177} \mathrm{Lu}$ are beta-emitters; they differ in maximum energy level (2.27 MeV versus $0.49 \mathrm{MeV}$ ), tissue penetration depth (11 mm versus $2 \mathrm{~mm}$ ) and half-life (2.67 days

Table 4 Follow-up recommendations under sunitinib therapy (ref. [48])

At baseline, at day 15 of therapy and monthly thereafter

$$
\begin{aligned}
& \text { - physical examination including blood pressure and heart rate } \\
& \text { - urine dipstick for proteinuria } \\
& \text { - complete blood count with differential } \\
& \text { - blood chemistry including creatinine, electrolytes, liver function }
\end{aligned}
$$

At baseline and three-monthly thereafter

- Thyroid stimulating hormone, T3, and T4 versus 6.68 days) [50]. As a gamma-ray emitter, ${ }^{177} \mathrm{Lu}$ can also be used for dosimetry and to monitor tumor response. Doselimiting toxicities are imposed by bone marrow and kidney irradiation. Therefore, the cumulative dose of radiolabelled SSA is fractionated in sequential cycles (usually 4-5), delivered systemically every 6-9 weeks.

Over the last 25 years, the antitumor effect of PRRT had been shown only in non-randomized early-phase studies, with quite similar efficacy for ${ }^{90} \mathrm{Y}$ and ${ }^{177} \mathrm{Lu}$ and disease control rates of $68-94 \%$ [51]. NETTER-1 is the first randomized phase III trial clearly demonstrating superiority of ${ }^{177} \mathrm{Lu}$-dotatate over high-dose octreotide LAR in octreoscan-positive midgut NETs, progressive on standard dose SSA [52]. After a median follow up of 14 months, a $79 \%$ reduction in the risk of progression or death was seen in the PRRT arm $(\mathrm{P}<0.0001$; HR 0.21 ; 95\% confidence interval 0.13-0.33). Median PFS was not reached in the investigational arm versus 8.4 months in the control arm. The overall response rate was $18 \%$ for ${ }^{177} \mathrm{Lu}$-dotatate versus $3 \%$ for high-dose octreotide LAR. PRRT is the only NET therapy with a distinct predictive biomarker, namely the baseline SSTR expression density (Krenning score). pNETs appear to respond better, but relapse earlier [53]. Large lesions, high hepatic tumor burden, fluorodeoxyglucose avidity and high Ki-67 are negative predictive factors $[51,54,55]$. The role of PRRT combinations with radiosensitizing cytotoxic agents or targeted agents, such as everolimus, remains to be further investigated in large randomized trials, as do treatment combinations of both radionucleotides aiming to take advantage of their different penetration ranges in targeting a variety of lesion types (large versus small size, heterogeneous SSRT expression, etc.).

Careful patient selection, appropriate timing of therapy, dose optimization and rigorous monitoring are mandatory to minimize the risk of short- and long-term toxicity, summarized in Table 6 [50,51,56-58]. ${ }^{177} \mathrm{Lu}$-dotatate has a more favorable toxicity profile, particularly concerning renal and hematological adverse events. Risk factors for increased toxicity after PRRT include the number of prior therapies, exposure to chemotherapy with alkylating agents, radiationbased therapy, age $>65$ years, impaired renal function, depleted myeloid reserve and poor performance status [58]. Normal age-adjusted renal function is mandatory for ${ }^{90} \mathrm{Y}$-labelled peptides. Mild renal impairment can be tolerated for ${ }^{177} \mathrm{Lu}-$ labeled peptides; however, glomerular filtration rate and tubular extraction rate should be at least $60 \%$ of mean ageadjusted normal values [59]. White blood cell count should be $>3000 / \mu \mathrm{L}$, with absolute neutrophil count $>1000 / \mu \mathrm{L}$, platelets $>75,000 / \mu \mathrm{L}$ for ${ }^{177} \mathrm{Lu}$ and $>90.000 / \mu \mathrm{L}$ for ${ }^{90} \mathrm{Y}$, and red blood cell count $>3 \times 10^{6} / \mu \mathrm{L}[59]$.

\section{Concluding remarks}

The therapeutic armamentarium for the treatment of GEPNETs has expanded significantly over the last two decades. However, the ideal sequencing strategy remains controversial. As patients live longer and remain active for a longer period of time, the optimal treatment toxicity management is of 
Table 5 Recommendations for clinical management of sunitinib toxicity

\begin{tabular}{|c|c|c|}
\hline General description/incidence (\%) & Preventive measures & Treatment \\
\hline $\begin{array}{l}\text { Fatigue }(32 \%) / \text { Asthenia }(34 \%) \\
\text { - rule out other causes (thyroid } \\
\text { dysfunction, psychological/emotional } \\
\text { distress, anemia, etc.) } \\
\text { - typically after } 1 \text { month; peak after } 2 \\
\text { to } 3 \text { months }\end{array}$ & $\begin{array}{l}\text { - ensure a consistent sleep cycle } \\
\text { - maintain activity levels during the } \\
\text { day } \\
\text { - avoid excessive caffeine and alcohol } \\
\text { - adequate fluid and nutritional intake }\end{array}$ & $\begin{array}{l}\text { - dose reductions do not have tremendous impact } \\
\text { - grade } 3 / 4 \text { : interrupt until recovery to } \leq \text { grade } 1 / 2 \\
\text { - consider intermittent dosing } 2 \text { weeks on } / 1 \text { week off }\end{array}$ \\
\hline $\begin{array}{l}\text { Hand-foot syndrome }(23 \%) \\
\text { - typically occurs within first } 2 \text { to } \\
4 \text { weeks }\end{array}$ & $\begin{array}{l}\text { - focus on prevention and patient } \\
\text { education } \\
\text { - inspection of palms and soles; treat } \\
\text { pre-existent calluses or hyperkeratotic } \\
\text { areas } \\
\text { - avoid exposure to hot water, } \\
\text { constrictive footwear or excessive skin } \\
\text { friction. } \\
\text { - use of cotton gloves and socks } \\
\text { - daily application of moisturizing } \\
\text { cream from day } 1\end{array}$ & $\begin{array}{l}\text { - topic antiseptic or antibiotic treatment as indicated } \\
\text { - keratolytics such as urea } 20-40 \% \text { or salicylic acid } 6 \% \\
\text { for hyperkeratotic areas } \\
\text { - topic or systemic analgesics } \\
\text { - grade } 3 / 4 \text { : interrupt until recovery to } \leq \text { grade } 1 / 2 \\
\text { - grade 3: reinitiate at same dose, unless recurrent } \\
\text { - grade } 4 \text { : reinitiate at lower dose or discontinue }\end{array}$ \\
\hline $\begin{array}{l}\text { Hypertension }(26 \%) \\
\text { - class effect of anti-angiogenics }\end{array}$ & $\begin{array}{l}\text { - actively screen for hypertension (may } \\
\text { include home monitoring) }\end{array}$ & $\begin{array}{l}\text { - treat as appropriate per standard guidelines } \\
\text { - angiotensin-converting enzyme inhibitors or } \\
\text { angiotensin II receptor blockers } \\
\text { - dihydropyridine calcium channel blockers } \\
\text { - grade 3/4: interrupt until recovery to } \leq \text { grade } 1 / 2 \\
\text { - grade 3: reinitiate at same dose, unless recurrent } \\
\text { - grade 4: reinitiate at lower dose or discontinue }\end{array}$ \\
\hline $\begin{array}{l}\text { Hypothyroidism }(7 \%) \\
\text { - may be proceeded by an episode of } \\
\text { hyperthyroidism }\end{array}$ & & $\begin{array}{l}\text { - thyroxine replacement therapy per standard guidelines } \\
\text { - sunitinib dose interruption or adjustment exceptional }\end{array}$ \\
\hline $\begin{array}{l}\text { Diarrhea }(59 \%) \\
\text { - rule out other causes (disease related, } \\
\text { pancreatic insufficiency, infection, } \\
\text { drugs, etc.) } \\
\text { - sunitinib diarrhea typically resolves a } \\
\text { few days after discontinuation }\end{array}$ & $\begin{array}{l}\text { - avoid caffeine, high lactose-containing, } \\
\text { fatty or high fibre foods and } \\
\text { fruits (except pectin-containing fruit) } \\
\text { - maintain adequate hydration }\end{array}$ & $\begin{array}{l}\text { - oral or intravenous (re)hydration as indicated } \\
\text { - oral anti-diarrheals (e.g., loperamide) } \\
\text { - grade 3/4: interrupt until recovery to } \leq \text { grade } 1 / 2 \\
\text { - grade 3: reinitiate at same dose, unless recurrent } \\
\text { - grade } 4 \text { : reinitiate at lower dose or discontinue }\end{array}$ \\
\hline $\begin{array}{l}\text { Hematologic toxicity } \\
\text { Thrombocytopenia (17\%) } \\
\text { Neutropenia }(29 \%)\end{array}$ & & $\begin{array}{l}\text { - grade } 3 / 4 \text { : interrupt until recovery to } \leq \text { grade } 2 \\
\text { - grade } 3 \text { : reinitiate at same dose, unless recurrent } \\
\text { - grade } 4 \text { : reinitiate at lower dose or discontinue }\end{array}$ \\
\hline $\begin{array}{l}\text { Nausea }(45 \%) / \text { vomiting }(34 \%) \\
\text { - rule out other causes than sunitinib }\end{array}$ & & $\begin{array}{l}\text { - early treatment with alizapride/metoclopramide } \\
\text { - caution with CYP3A4 interaction (e.g., ondansetron) }\end{array}$ \\
\hline Stomatitis (22\%) & $\begin{array}{l}\text { - identical to table } 3 \text { (everolimus } \\
\text { associated stomatitis) }\end{array}$ & $\begin{array}{l}\text { - dose reductions rarely necessary for isolated oral } \\
\text { mucositis, may be considered for multiple toxicities } \\
\text { - grade 3/4: interrupt until recovery to } \leq \text { grade } 1 / 2 \\
\text { - grade 3: reinitiate at same dose, unless recurrent } \\
\text { - grade 4: reinitiate at lower dose or discontinue }\end{array}$ \\
\hline
\end{tabular}

paramount importance. Although clinical trials mainly focus on grade 3-4 toxicity, even persisting grade 2 toxicity becomes more of an issue, impacting quality of life and compliance in this setting of long-term treatment. Nonetheless, prospective data on optimal adverse event management are lacking and recommendations are largely based on expert opinion and drug prescribing information. When interpreting the available evidence in the literature, one should pay attention to the version of the Common Terminology Criteria for Adverse Events used, as historically used versions might differ substantially from current ones and may lead to under- or over-reporting.
Little is known about the exact magnitude of the impact of dose interruptions and/or modifications of these drugs in the GEPNET indication. The predictive significance of adverse event occurrence for treatment outcome has been demonstrated for epidermal growth factor receptor inhibitorrelated skin toxicity in metastatic colorectal cancer and certain MTKI-related toxicity in advanced RCC. However, this information is lacking in the setting of NET treatment and needs to be systematically addressed in future clinical trials, as does the need for toxicity-related biomarkers in general. 
Table 6 Recommendations for clinical management of peptide receptor radionucleotide therapy (PRRT) toxicit

\begin{tabular}{|c|c|c|}
\hline General description & Preventive measures & Treatment \\
\hline \multicolumn{3}{|l|}{ Acute toxicity } \\
\hline $\begin{array}{l}\text { Nausea/vomiting } \\
\text { - usually mild and self-limiting } \\
\text { - primarily attributable to the concomitant } \\
\text { amino acid infusion given for } \\
\text { nephroprotective purposes }\end{array}$ & & - treatment with standard anti-emetic therapy \\
\hline $\begin{array}{l}\text { Carcinoid crisis }(<1 \%) \\
\text { - due to massive release of metabolically active } \\
\text { amines }\end{array}$ & & $\begin{array}{l}\text { - octreotide } 0.5 \mathrm{mg} \text { subcutaneous, t.i.d. } \\
\text { - adrenergic drugs should be avoided in case } \\
\text { of shock } \\
\text { - octreotide up to } 1 \mathrm{mg} \text { intravenous in case of } \\
\text { shock }\end{array}$ \\
\hline \multicolumn{3}{|l|}{$\begin{array}{l}\text { Hematologic toxicity } \\
\text { - due to bone marrow irradiation } \\
\text { - usually mild and self-limiting } \\
\text { - occurring typically } 4-6 \text { weeks after } \\
\text { administration } \\
\text { - lymphopenia most frequent }\end{array}$} \\
\hline \multicolumn{3}{|l|}{$\begin{array}{l}\text { Hepatotoxicity } \\
\text { - due to hepatocyte } \\
\text { irradiation (radiation-induced liver disease) } \\
\text { - theoretically risk is increased in high liver } \\
\text { burden, although not well documented }\end{array}$} \\
\hline \multicolumn{3}{|l|}{ Long-term toxicity } \\
\hline $\begin{array}{l}\text { Renal toxicity } \\
\text { - radionucleotides are reabsorbed in the } \\
\text { proximal tubules and accumulate in the } \\
\text { renal interstitium, causing inflammation and } \\
\text { fibrosis leading to irreversible kidney injury } \\
\text { - coadministration of positively charged } \\
\text { amino acids competitively inhibits proximal } \\
\text { tubular radiopeptide reabsorption and } \\
\text { recirculation in the kidney and decreases } \\
\text { radioactive uptake in the kidneys by } 40 \%\end{array}$ & $\begin{array}{l}\text { - use of appropriate dosimetry } \\
\text { - } 25 \mathrm{~g} \text { lysine and } 25 \mathrm{~g} \text { arginine diluted } \\
\text { in } 2 \mathrm{~L} \text { saline solution, started } 30-60 \mathrm{~min} \\
\text { before PRRT and maintained over } 4 \mathrm{~h} \\
\text { - consider dose reduction in } \\
\text { case of pre-existing nephrotoxic } \\
\text { factors (uncontrolled diabetes, } \\
\text { hypertension, old age, single kidney, etc.) }\end{array}$ & $\begin{array}{l}\text { - overall a mild loss of renal function over time } \\
\text { occurs of } 7.3 \% / \text { year for yttrium- } 90\left({ }^{90} \mathrm{Y}\right) \text { and } \\
3.8 \% / \text { year for lutetium-177 }\left({ }^{177} \mathrm{Lu}\right) \text {. } \\
\text { - incidence grade } 3 / 4 \text { renal toxicity in }{ }^{90} \mathrm{Y}: 3-9 \% \\
\text { - incidence grade } 4 \text { renal toxicity in }{ }^{177} \mathrm{Lu} \text { : } \\
<0.4 \% \\
\text { - end-stage renal failure after PRRT is } \\
\text { extremely rare }\end{array}$ \\
\hline $\begin{array}{l}\text { Leukemia/myelodysplastic syndrome } \\
\text { - occurring in }<2 \% \text {, typically } 5-10 \text { years after } \\
\text { therapy }\end{array}$ & & \\
\hline
\end{tabular}

Finally, we want to stress the critical role of patient and treating physician in maintaining adequate dose intensity in GEPNET medical treatment. The diversity and complexity of NET management today requires the support of a dedicated multidisciplinary team, e.g., including an oncologist, gastroenterologist, dermatologist, cardiologist, psychologist, nutritional expert, and oncology nurses.

\section{References}

1. Gatta G, van der Zwan JM, Casali PG, et al; RARECARE working group. Rare cancers are not so rare: the rare cancer burden in Europe. Eur J Cancer 2011;47:2493-2511.

2. Fraenkel M, Kim M, Faggiano A, de Herder WW, Valk GD; Knowledge NETwork. Incidence of gastroenteropancreatic neuroendocrine tumours: a systematic review of the literature. Endocr Relat Cancer 2014;21:R153-R163.
3. Frilling A, Akerström G, Falconi $\mathrm{M}$, et al. Neuroendocrine tumor disease: an evolving landscape. Endocr Relat Cancer 2012;19:R163-R185.

4. Lloyd RV, Osamura RY, Klöppel G, J R, eds. WHO classification of tumours of endocrine organs. WHO/IARC Classification of Tumours, $4^{\text {th }}$ Edition, Volume 10. IARC Publications: Lyon; 2017.

5. de Hosson LD, Veenendaal LMV, Schuller Y, et al. Clinical benefit of systemic treatment in patients with advanced pancreatic and gastro-intestinal neuroendocrine tumours according to ESMOMCBS and ASCO framework. Ann Oncol 2017 Sep 26 [Epub ahead of print]. doi:10.1093/annonc/mdx547.

6. Rinke A, Krug S. Neuroendocrine tumours - Medical therapy: Biological. Best Pract Res Clin Endocrinol Metab 2016;30:79-91.

7. Hankus J, Tomaszewska R. Neuroendocrine neoplasms and somatostatin receptor subtypes expression. Nucl Med Rev Cent East Eur 2016;19:111-117.

8. Khan MS, El-Khouly F, Davies P, Toumpanakis C, Caplin ME. Long-term results of treatment of malignant carcinoid syndrome with prolonged release Lanreotide (Somatuline Autogel). Aliment Pharmacol Ther 2011;34:235-242. 
9. Kvols LK, Moertel CG, O'Connell MJ, Schutt AJ, Rubin J, Hahn RG. Treatment of the malignant carcinoid syndrome. Evaluation of a long-acting somatostatin analogue. NEngl JMed 1986;315:663-666.

10. Rubin J, Ajani J, Schirmer W, et al. Octreotide acetate long-acting formulation versus open-label subcutaneous octreotide acetate in malignant carcinoid syndrome. J Clin Oncol 1999;17:600-606.

11. Ruszniewski P, Ducreux M, Chayvialle JA, et al. Treatment of the carcinoid syndrome with the longacting somatostatin analogue lanreotide: a prospective study in 39 patients. Gut 1996;39:279-283.

12. Pokuri VK, Fong MK, Iyer R. Octreotide and lanreotide in gastroenteropancreatic neuroendocrine tumors. Curr Oncol Rep 2016;18:7.

13. Öberg K, Lamberts SW. Somatostatin analogues in acromegaly and gastroenteropancreatic neuroendocrine tumours: past, present and future. Endocr Relat Cancer 2016;23:R551-R566.

14. Rinke A, Müller HH, Schade-Brittinger C, et al; PROMID Study Group. Placebo-controlled, double-blind, prospective, randomized study on the effect of octreotide LAR in the control of tumor growth in patients with metastatic neuroendocrine midgut tumors: a report from the PROMID Study Group. J Clin Oncol 2009;27:4656-4663.

15. Rinke A, Wittenberg M, Schade-Brittinger C, et al; PROMID Study Group. Placebo-controlled, double-blind, prospective, randomized study on the effect of octreotide LAR in the control of tumor growth in patients with metastatic neuroendocrine midgut tumors (PROMID): results of long-term survival. Neuroendocrinology 2017;104:26-32.

16. Caplin ME, Pavel M, Ćwikła JB, et al; CLARINET Investigators. Lanreotide in metastatic enteropancreatic neuroendocrine tumors. N Engl J Med 2014;371:224-233.

17. Trendle MC, Moertel CG, Kvols LK. Incidence and morbidity of cholelithiasis in patients receiving chronic octreotide for metastatic carcinoid and malignant islet cell tumors. Cancer 1997;79:830-834.

18. Strosberg JR, Fisher GA, Benson AB, et al; GEPNET Treatment Consensus Panel. Systemic treatment in unresectable metastatic well-differentiated carcinoid tumors: consensus results from a modified delphi process. Pancreas 2013;42:397-404.

19. Broder MS, Beenhouwer D, Strosberg JR, Neary MP, Cherepanov D. Gastrointestinal neuroendocrine tumors treated with high dose octreotide-LAR: a systematic literature review. World J Gastroenterol 2015;21:1945-1955.

20. Wolin EM, Jarzab B, Eriksson B, et al. Phase III study of pasireotide long-acting release in patients with metastatic neuroendocrine tumors and carcinoid symptoms refractory to available somatostatin analogues. Drug Des Devel Ther 2015;9:5075-5086.

21. Mirvis E, Mandair D, Garcia-Hernandez J, Mohmaduvesh M, Toumpanakis C, Caplin M. Role of interferon-alpha in patients with neuroendocrine tumors: a retrospective study. Anticancer Res 2014;34:6601-6607.

22. Pavel M, O’Toole D, Costa F, et al; Vienna Consensus Conference participants. ENETS Consensus guidelines update for the management of distant metastatic disease of intestinal, pancreatic, bronchial neuroendocrine neoplasms (NEN) and NEN of unknown primary site. Neuroendocrinology 2016;103:172-185.

23. Hauschild A, Kähler KC, Schäfer M, Fluck M. Interdisciplinary management recommendations for toxicity associated with interferon-alfa therapy. J Dtsch Dermatol Ges 2008;6:829-837.

24. Daud A, Soon C, Dummer R, et al. Management of pegylated interferon alpha toxicity in adjuvant therapy of melanoma. Expert Opin Biol Ther 2012;12:1087-1099.

25. Rubin KM, Vona K, Madden K, McGettigan S, Braun IM. Side effects in melanoma patients receiving adjuvant interferon alfa-2b therapy: a nurse's perspective. Support Care Cancer 2012;20:1601-1611.

26. Ricci S, Antonuzzo A, Galli L, et al. Long-acting depot lanreotide in the treatment of patients with advanced neuroendocrine tumors.
Am J Clin Oncol 2000;23:412-415.

27. Markham A. Telotristat ethyl: first global approval. Drugs 2017;77:793-798.

28. Horsch D, Kulke MH, Caplin M, et al. Efficacy and safety of telotristat etiprate in patients with carcinoid syndrome not adequately controlled by somatostatin analogue therapy: analysis of the ongoing TELESTAR extension period. ENETS $13^{\text {th }}$ Annual Conference, Barcelona, Spain 2016 Mar 9-11. Available from: https://www.enets.org/13 ${ }^{\text {th }}$ _annual_conference_barcelona_2016. html.

29. Kulke MH, Hörsch D, Caplin ME, et al. Telotristat ethyl, a tryptophan hydroxylase inhibitor for the treatment of carcinoid syndrome. J Clin Oncol 2017;35:14-23.

30. Pavel M, Benavent M, Perros P, et al. Telotristat ethyl in carcinoid syndrome: safety and efficacy results of an open-label extension of the TELECAST phase 3 clinical trial [abstract no. 1942 plus poster]. ENETS $13^{\text {th }}$ Annual Conference, Barcelona, Spain 2017.

31. Pavel M, Gross D, Benavent M, et al. Efficacy and safety results of telotristat ethyl in patients with carcinoid syndrome during the double - blind treatment period of the TELECAST phase 3 clinical trial. North American Neuroendocrine Tumor Society Annual Symposium 2016, Jackson, Wyoming 2016 Sep 30-0ct 1 . Available from: https://www.nanets.net/nanets_cd/2016/pdfs/C8.pdf.

32. Lamarca A, Barriuso J, McNamara MG, Hubner RA, Valle JW. Telotristat ethyl: a new option for the management of carcinoid syndrome. Expert Opin Pharmacother 2016;17:2487-2498.

33. Yao JC, Shah MH, Ito T, et al; RAD001 in Advanced Neuroendocrine Tumors, Third Trial (RADIANT-3) Study Group. Everolimus for advanced pancreatic neuroendocrine tumors. $N$ Engl J Med 2011;364:514-523.

34. Yao JC, Fazio N, Singh S, et al; RAD001 in Advanced Neuroendocrine Tumours, Fourth Trial (RADIANT-4) Study Group. Everolimus for the treatment of advanced, non-functional neuroendocrine tumours of the lung or gastrointestinal tract (RADIANT-4): a randomised, placebo-controlled, phase 3 study. Lancet 2016;387:968-977.

35. Pavel ME, Hainsworth JD, Baudin E, et al; RADIANT-2 Study Group. Everolimus plus octreotide long-acting repeatable for the treatment of advanced neuroendocrine tumours associated with carcinoid syndrome (RADIANT-2): a randomised, placebocontrolled, phase 3 study. Lancet 2011;378:2005-2012.

36. Afinitor (everolimus). Summary of product characteristics. Novartis Pharma AG, 2016.

37. Aapro M, Andre F, Blackwell K, et al. Adverse event management in patients with advanced cancer receiving oral everolimus: focus on breast cancer. Ann Oncol 2014;25:763-773.

38. Grünwald V, Weikert S, Pavel ME, et al. Practical management of everolimus-related toxicities in patients with advanced solid tumors. Onkologie 2013;36:295-302.

39. Martins F, de Oliveira MA, Wang Q, et al. A review of oral toxicity associated with mTOR inhibitor therapy in cancer patients. Oral Oncol 2013;49:293-298.

40. Peterson DE, O’Shaughnessy JA, Rugo HS, et al. Oral mucosal injury caused by mammalian target of rapamycin inhibitors: emerging perspectives on pathobiology and impact on clinical practice. Cancer Med 2016;5:1897-1907.

41. Willemsen AE, Grutters JC, Gerritsen WR, van Erp NP, van Herpen CM, Tol J. mTOR inhibitor-induced interstitial lung disease in cancer patients: Comprehensive review and a practical management algorithm. Int J Cancer 2016;138:2312-2321.

42. Ravaud A, Urva SR, Grosch K, Cheung WK, Anak O, Sellami DB. Relationship between everolimus exposure and safety and efficacy: meta-analysis of clinical trials in oncology. Eur J Cancer 2014;50:486-495.

43. Faivre S, Niccoli P, Castellano D, et al. Sunitinib in pancreatic neuroendocrine tumors: updated progression-free survival and 
final overall survival from a phase III randomized study. Ann Oncol 2017;28:339-343.

44. Raymond E, Dahan L, Raoul JL, et al. Sunitinib malate for the treatment of pancreatic neuroendocrine tumors. $N$ Engl J Med 2011;364:501-513

45. Houk BE, Bello CL, Poland B, Rosen LS, Demetri GD, Motzer RJ. Relationship between exposure to sunitinib and efficacy and tolerability endpoints in patients with cancer: results of a pharmacokinetic/pharmacodynamic meta-analysis. Cancer Chemother Pharmacol 2010;66:357-371.

46. Lipworth AD, Robert C, Zhu AX. Hand-foot syndrome (handfoot skin reaction, palmar-plantar erythrodysesthesia): focus on sorafenib and sunitinib. Oncology 2009;77:257-271.

47. Kollmannsberger C. Sunitinib side effects as surrogate biomarkers of efficacy. Can Urol Assoc J 2016;10:S245-S247.

48. Sutent (sunitinib). Summary of product characteristics (centralized license), applicable to all countries of the EU and Norway [V: Date of revision of text 11/2016; LC].

49. Valle JW, Faivre S, Hubner RA, Grande E, Raymond E. Practical management of sunitinib toxicities in the treatment of pancreatic neuroendocrine tumors. Cancer Treat Rev 2014;40:1230-1238.

50. Cives M, Strosberg J. Radionuclide therapy for neuroendocrine tumors. Curr Oncol Rep 2017;19:9.

51. Bodei L, Kwekkeboom DJ, Kidd M, Modlin IM, Krenning EP. Radiolabeled somatostatin analogue therapy of gastroenteropancreatic cancer. Semin Nucl Med 2016;46:225-238.

52. Strosberg J, El-Haddad G, Wolin E, et al; NETTER-1 Trial
Investigators. Phase 3 trial of $177 \mathrm{Lu}$-dotatate for midgut neuroendocrine tumors. N Engl J Med 2017;376:125-135.

53. Kwekkeboom DJ, Teunissen JJ, Bakker WH, et al. Radiolabeled somatostatin analog [177Lu-DOTA0,Tyr3]octreotate in patients with endocrine gastroenteropancreatic tumors. J Clin Oncol 2005;23:2754-2762.

54. Ezziddin S, Attassi M, Yong-Hing CJ, et al. Predictors of long-term outcome in patients with well-differentiated gastroenteropancreatic neuroendocrine tumors after peptide receptor radionuclide therapy with $177 \mathrm{Lu}$-octreotate. J Nucl Med 2014;55:183-190.

55. Severi S, Grassi I, Nicolini S, Sansovini M, Bongiovanni A, Paganelli G. Peptide receptor radionuclide therapy in the management of gastrointestinal neuroendocrine tumors: efficacy profile, safety, and quality of life. Onco Targets Ther 2017;10:551-557.

56. Brabander T, Teunissen JJ, Van Eijck CH, et al. Peptide receptor radionuclide therapy of neuroendocrine tumours. Best Pract Res Clin Endocrinol Metab 2016;30:103-114.

57. Erbas B, Tuncel M. Renal function assessment during peptide receptor radionuclide therapy. Semin $\mathrm{Nucl}$ Med 2016;46:462-478.

58. Kesavan M, Turner JH. Myelotoxicity of peptide receptor radionuclide therapy of neuroendocrine tumors: a decade of experience. Cancer Biother Radiopharm 2016;31:189-198.

59. Bodei L, Mueller-Brand J, Baum RP, et al. The joint IAEA, EANM, and SNMMI practical guidance on peptide receptor radionuclide therapy (PRRNT) in neuroendocrine tumours. Eur J Nucl Med Mol Imaging 2013;40:800-816. 